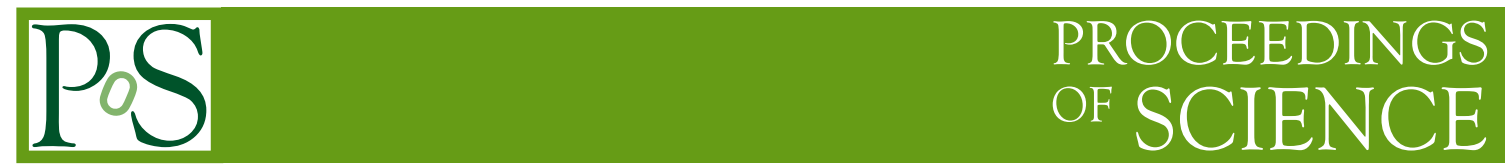

\title{
Charmless B decays
}

\section{Brais Sanmartin Sedes ${ }^{\dagger}$}

Universidade de Santiago de Compostela (ES)

E-mail: brais.sanmartin. sedesecern.ch

Charmless B meson decays proceed via suppressed $b \rightarrow u$ tree and $b \rightarrow s, d$ penguin diagrams and are thus sensitive to New Physics. We present recent results from the LHCb experiment on angular analyses of decays of $B$ mesons to a pair of light vector mesons $(B \rightarrow V V)$ and searches for very suppressed decay modes.

The European Physical Society Conference on High Energy Physics

22-29 July 2015

Vienna, Austria

* Speaker.

${ }^{\dagger}$ on behalf of the LHCb collaboration. 


\section{Introduction}

Hadronic decays of beauty hadrons into final states without charm quarks (charmless decays) are suppressed in the Standard Model (SM) of elementary particles. They proceed predominantly through $b \rightarrow u$ transitions, mediated by the emission of a virtual $W^{ \pm}$boson, and $b \rightarrow s, d$ transitions, mediated by the exchange of a virtual $W^{ \pm}$boson and a virtual quark. The respective "tree" and "penguin" amplitudes are of similar size, allowing for possible large quantum interference effects measurable as charge-parity $(C P)$ violating asymmetries. New particles not described in the SM may contribute with additional amplitudes, and therefore affect both the decay rates and the $C P$ asymmetries. In this work, the most recent results from the LHCb experiment on angular analyses of $B \rightarrow V V$ decays and searches for very supressed decay modes are presented.

\section{2. $C P$ asymmetries and polarisation fractions in $B_{s}^{0} \rightarrow K^{* 0} \bar{K}^{* 0}[1]$}

The $B_{s}^{0} \rightarrow K^{* 0} \bar{K}^{* 0}$ decay is mediated by a $b \rightarrow s d \bar{d}$ flavour-changing neutral current (FCNC) transition, which in the SM proceeds through loop diagrams at leading order. This decay, when considered in association with its U-spin symmetric channel $B^{0} \rightarrow K^{* 0} \bar{K}^{* 0}$, can be a possible field for precision tests of the SM predictions [2, 3, 4]. When interference with a final state containing a scalar meson is considered, in addition to the vector-vector meson states, six independent helicity amplitudes contribute [5].

The $B_{s}^{0} \rightarrow K^{* 0} \bar{K}^{* 0}$ decay was first observed with $35 \mathrm{pb}^{-1}$ of LHCb data [6]. A remarkably low longitudinal polarisation fraction was observed, at variance with that observed in the mirror channel $B^{0} \rightarrow K^{* 0} \bar{K}^{* 0}$ [7] and with some predictions from QCD factorisation [8, 9].

In the presented analysis, a search for non-SM electroweak amplitudes is reported in the decay $B_{s}^{0} \rightarrow K^{+} \pi^{-} K^{-} \pi^{+}$, with the $K \pi$ mass close to the $K^{*}(892)^{0}$ resonance $\left(\left|M(K \pi)-m_{K^{*}(892)^{0}}\right|<\right.$ $150 \mathrm{MeV} / \mathrm{c}^{2}$ ), through the measurement of all $C P$-violating observables accessible when the flavour of the bottom-strange meson is not identified. These observables include triple products (TPs) and other $C P$-odd quantities [10], many of which have not yet been measured in this decay mode. These studies are performed using $1.0 \mathrm{fb}^{-1}$ of $p p$ collision data taken by LHCb in 2011 at a centre-ofmass energy of $\sqrt{s}=7 \mathrm{TeV}$.

An extended unbinned maximum likelihood fit to the mass spectrum of the selected $B_{s}^{0} \rightarrow$ $K^{+} \pi^{-} K^{-} \pi^{+}$candidates is performed. A total of $697 \pm 31 B_{s}^{0} \rightarrow K^{+} \pi^{-} K^{-} \pi^{+}$candidates is obtained. The magnitudes and phases of the various amplitudes contributing to the $B_{s}^{0} \rightarrow K^{+} \pi^{-} K^{-} \pi^{+}$ decay are determined using a five-dimensional fit to the three helicity angles, ${ }^{1}$ and to the invariant mass of the two $K \pi$ pairs of all candidates with a four-body invariant mass $\mid M\left(K^{+} \pi^{-} K^{-} \pi^{+}\right)-$ $m_{B_{s}^{0}} \mid<30 \mathrm{MeV} / \mathrm{c}^{2}$ (Fig. 1).

The low polarisation of the vector-vector decay is confirmed by the measurement $f_{L}=0.201 \pm$ 0.057 (stat.) \pm 0.040 (syst.), and a large S-wave contribution is found.

${ }^{1} \Omega \equiv\left\{\theta_{1}, \theta_{2}, \varphi\right\}$, where $\theta_{1(2)}$ is the angle between the direction of $K^{+(-)}$meson and the direction opposite to the $B$-meson momentum in the rest frame of $V_{1(2)}$ and $\varphi$ is the angle between the decay planes of the two vector mesons in the $B$-meson rest frame. 

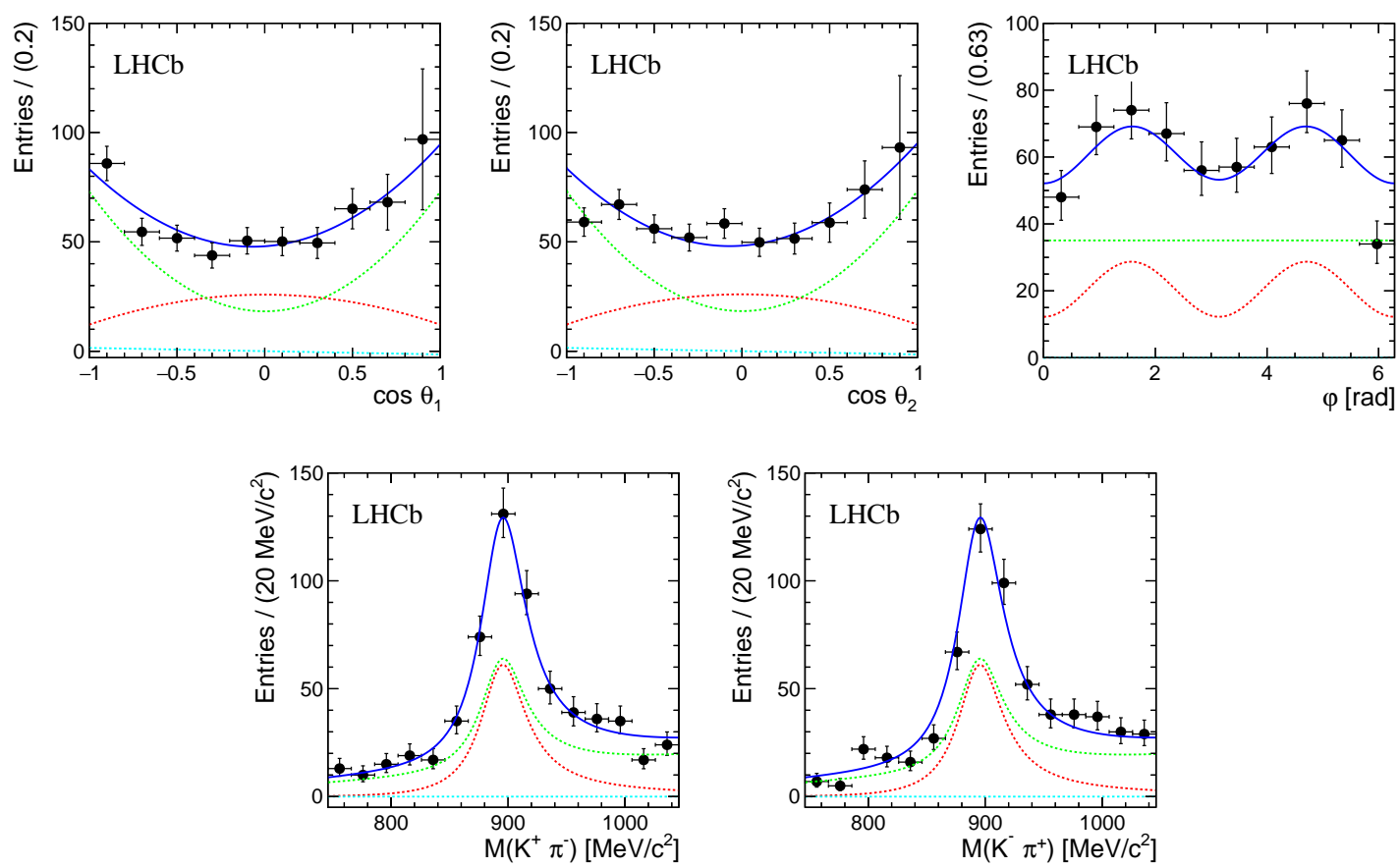

Figure 1: Results of the simultaneous fit to $B_{s}^{0} \rightarrow K^{+} \pi^{-} K^{-} \pi^{+}$candidates (blue solid line) in the three helicity angles (top) and in the invariant mass of $K \pi$ pairs (bottom). The points represent the data after background subtraction and acceptance correction. The red dashed line is the P-wave component, the green dashed line is the $\mathrm{S}$-wave component and the light-blue dashed line represents the interference term between the $C P$-odd component of the $\mathrm{S}$-wave and the longitudinal component of the $\mathrm{P}$-wave.

A test of the $\mathrm{SM}$ is performed by measuring eight $C P$-violating quantities which are predicted to be small in the SM. The measured asymmetries are found to be compatible with the SM expectation, within 2 standard deviations.

Finally, an update of the $B_{s}^{0} \rightarrow K^{* 0} \bar{K}^{* 0}$ branching fraction, using the $B^{0} \rightarrow \phi K^{* 0}$ decay as normalisation channel, yields $\mathscr{B}\left(B_{s}^{0} \rightarrow K^{* 0} \bar{K}^{* 0}\right)=(10.8 \pm 2.1$ (stat. $) \pm 1.4$ (syst. $\left.) \pm 0.6\left(f_{d} / f_{s}\right)\right) \times$ $10^{-6}$, in agreement with the theoretical prediction [8].

\section{Amplitude analysis of $B^{0} \rightarrow \rho^{0} \rho^{0}[11]$}

The study of $B$ meson decays to $\rho \rho$ final states provides the most powerful constraint to date for the Cabibbo-Kobayashi-Maskawa (CKM) angle $\alpha \equiv \arg \left[\left(V_{t d} V_{t b}^{*}\right) /\left(V_{u d} V_{u b}^{*}\right)\right][12,13,14]$. Measurements of the $B^{0} \rightarrow \rho^{0} \rho^{0}$ branching fraction and longitudinal polarisation fraction at $\mathrm{LHCb}$ can be used as inputs in the determination of $\alpha[13,14]$. The BaBar and Belle experiments reported previous evidence for the $B^{0} \rightarrow \rho^{0} \rho^{0}$ decay $[15,16]$. Each experiment measured the fraction $\mathrm{f}_{\mathrm{L}}$ of decays yielding a longitudinally polarised final state through an angular analysis. These results differ at the level of 2.0 standard deviations. In addition, Belle reported a hint of the $B^{0} \rightarrow$ $\rho^{0} f_{0}(980)$ decay [16].

The search of the $B^{0} \rightarrow \rho^{0} \rho^{0}$ decay is performed using $1.0 \mathrm{fb}^{-1}$ of $p p$ collision data taken by $\mathrm{LHCb}$ in 2011 at a centre-of-mass energy of $\sqrt{s}=7 \mathrm{TeV}$ and $2.0 \mathrm{fb}^{-1}$ in 2012 at a centre-of-mass 
energy of $\sqrt{s}=8 \mathrm{TeV}$. A yield of $634 \pm 28$ (stat.) \pm 8 (syst.) $B^{0} \rightarrow\left(\pi^{+} \pi^{-}\right)\left(\pi^{+} \pi^{-}\right)$signal decays with $\pi^{+} \pi^{-}$pairs in the $300-1100 \mathrm{MeV} / \mathrm{c}^{2}$ mass range is obtained. In the same $\pi^{+} \pi^{-}$pairs mass range, $B_{s}^{0} \rightarrow\left(\pi^{+} \pi^{-}\right)\left(\pi^{+} \pi^{-}\right)$decays are also observed with a statistical significance of more than 10 standard deviations. Even though the $B^{0} \rightarrow \rho^{0} \rho^{0}$ is expected to dominate the $\left(\pi^{+} \pi^{-}\right)$mass spectrum, the $\left(\pi^{+} \pi^{-}\right)$combinations can actually emerge from S-wave non-resonant and resonant contributions or other P- or D-wave resonances interfering with the signal. Hence, the determination of the $B^{0} \rightarrow \rho^{0} \rho^{0}$ yields requires a two-body mass and angular analysis $\left(M\left(\pi^{+} \pi^{-}\right)_{1,2}, \cos \theta_{1,2}\right.$ and $\varphi),{ }^{2}$ from which the fraction of the longitudinally polarised final state can be measured (Fig. 2).

The resulting longitudinal polarisation fraction of the $B^{0} \rightarrow \rho^{0} \rho^{0}$ decay is found to be $\mathrm{f}_{\mathrm{L}}=$ $0.745_{-0.058}^{+0.048}$ (stat.) \pm 0.034 (syst.). The value of the measured $B^{0} \rightarrow \rho^{0} \rho^{0}$ branching fraction, using the $B^{0} \rightarrow \phi K^{*}(892)^{0}$ decay as reference, is determined to be $\mathscr{B}\left(B^{0} \rightarrow \rho^{0} \rho^{0}\right)=(0.94 \pm 0.17$ (stat. $) \pm$ 0.09 (syst.) $\pm 0.06(\mathrm{BF})) \times 10^{-6}$, where the last uncertainty is due to the normalisation channel. This decay mode is observed for the first time with a significance of 7.1 standard deviations. The measured longitudinal polarisation fraction is consistent with the measured value from BaBar [15] while it differs by 2.3 standard deviations from the value obtained by Belle [16]. The branching fraction measurement is in agreement with the values measured by both BaBar [15] and Belle [16] collaborations.

The evidence of the $B^{0} \rightarrow \rho^{0} f_{0}(980)$ decay mode reported by the Belle collaboration [16] is not confirmed, and an upper limit at $90 \%$ confidence level is established $\mathscr{B}\left(B^{0} \rightarrow \rho^{0} f_{0}(980)\right) \times$ $\mathscr{B}\left(f_{0}(980) \rightarrow \pi^{+} \pi^{-}\right)<0.81 \times 10^{-6}$.
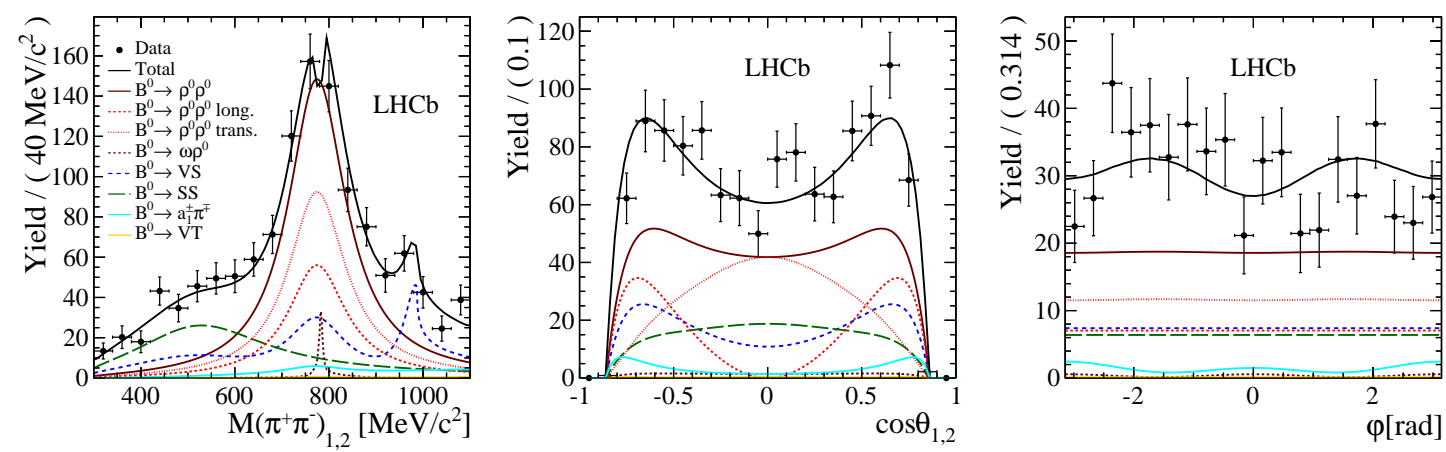

Figure 2: Background-subtracted $M\left(\pi^{+} \pi^{-}\right)_{1,2}, \cos \theta_{1,2}$ and $\varphi$ distributions. The black dots correspond to the four-body background-subtracted data and the black line is the projection of the fit model. The specific decays $B^{0} \rightarrow \rho^{0} \rho^{0}$ (brown), $B^{0} \rightarrow \omega \rho^{0}$ (dashed brown), $B^{0} \rightarrow V S$ (dashed blue), $B^{0} \rightarrow S S$ (long dashed green), $B^{0} \rightarrow V T$ (orange) and $B^{0} \rightarrow a_{1}^{ \pm} \pi^{\mp}$ (light blue) are also displayed. The $B^{0} \rightarrow \rho^{0} \rho^{0}$ contribution is split into longitudinal (dashed red) and transverse (dotted red) components. Interference contributions are only plotted for the total (black) model.

\section{First observation of $B_{s}^{0} \rightarrow \eta^{\prime} \eta^{\prime}[17]$}

The first observation of the $B_{s}^{0} \rightarrow \eta^{\prime} \eta^{\prime}$ decay is reported. The study is performed using $1.0 \mathrm{fb}^{-1}$

\footnotetext{
${ }^{2} \theta_{1(2)}$ is the angle between the direction of $\pi_{1(2)}^{-}$meson and the direction opposite to the $B$-meson momentum in the rest frame of $\rho_{1(2)}^{0}$, and $\varphi$ is the angle between the decay planes of the two vector mesons in the $B$-meson rest frame. The subscripts 1 and 2 refer to the first and second $\pi^{+} \pi^{-}$pairs, which are sorted randomly.
} 
of $p p$ collision data taken by LHCb in 2011 at a centre-of-mass energy of $\sqrt{s}=7 \mathrm{TeV}$ and $2.0 \mathrm{fb}^{-1}$ in 2012 at a centre-of-mass energy of $\sqrt{s}=8 \mathrm{TeV}$. The decay $B_{s}^{0} \rightarrow \eta^{\prime} \eta^{\prime}$ is expected to have a relatively large branching fraction $[18,19,20,21,22]$. The $\eta^{\prime} \eta^{\prime}$ final state is a pure $C P$ eigenstate. Decays to this final state of $B_{s}^{0}$ and $\bar{B}_{s}^{0}$ mesons flavour tagged at production may therefore be used to investigate time-dependent $C P$ asymmetries in a complementary way to the measurements in $B_{s}^{0} \rightarrow \phi \phi$, but without the need for an angular analysis.

The branching fraction is measured using the known $B^{ \pm} \rightarrow \eta^{\prime} K^{ \pm}$and $B^{ \pm} \rightarrow \phi K^{ \pm}$decays as calibration channels. The $\mathrm{CP}$ asymmetries of the calibration channels are also measured, relative to that of the $B^{ \pm} \rightarrow J / \psi K^{ \pm}$channel.

The $B_{s}^{0} \rightarrow \eta^{\prime} \eta^{\prime}$ signal yield is determined from a multidimensional unbinned extended maximum likelihood fit to the sample of $B_{s}^{0} \rightarrow \eta^{\prime} \eta^{\prime}$ (Fig. 3) and $B^{ \pm} \rightarrow \eta^{\prime} K^{ \pm}$candidates. We observe $36.4 \pm 7.8$ (stat.) \pm 1.6 (syst.) $B_{s}^{0} \rightarrow \eta^{\prime} \eta^{\prime}$ decays, corresponding to a significance of 6.4 standard deviations, including both statistical and systematic uncertainties.

The measured $B_{s}^{0} \rightarrow \eta^{\prime} \eta^{\prime}$ branching fraction, $\mathscr{B}\left(B_{s}^{0} \rightarrow \eta^{\prime} \eta^{\prime}\right)=[3.31 \pm 0.64$ (stat.) \pm 0.28 (syst.) \pm $0.12($ norm $)] \times 10^{-5}$, agrees with the theoretical predictions.

The $B^{ \pm} \rightarrow \eta^{\prime} K^{ \pm}$and $B^{ \pm} \rightarrow \phi K^{ \pm}$charge asymmetries are determined using the strategy adopted in Ref. [23] and are measured to be $A^{C P}\left(B^{ \pm} \rightarrow \eta^{\prime} K^{ \pm}\right)=[-0.2 \pm 1.2$ (stat.) \pm 0.1 (syst.) \pm 0.6 (norm.) $] \times$ $10^{-2}$ and $A^{C P}\left(B^{ \pm} \rightarrow \phi K^{ \pm}\right)=[+1.7 \pm 1.1$ (stat.) \pm 0.2 (syst.) \pm 0.6 (norm.) $) \times 10^{-2}$. These results are compatible with the hypothesis of $C P$ symmetry and with the SM predictions [24, 25].

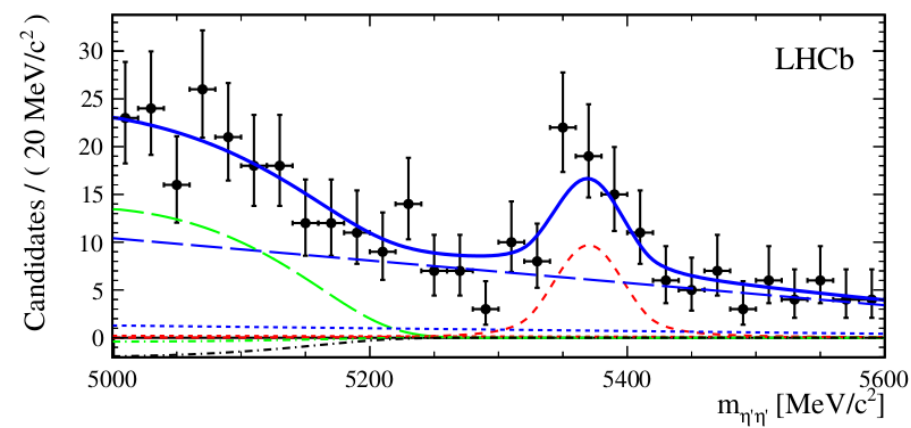

Figure 3: Distribution of the $\eta^{\prime} \eta^{\prime}$ invariant mass for the $B_{s}^{0} \rightarrow \eta^{\prime} \eta^{\prime}$ candidates with fit results overlaid. The components are the following: (dashed red curves) $B_{s}^{0} \rightarrow \eta^{\prime} \eta^{\prime}$ signal, (long-dashed blue curves) combinatorial background without an $\eta^{\prime}$ resonance in the final state, (dot-long-dashed black curves) partially reconstructed background without an $\eta^{\prime}$ resonance, (short-dashed red, short-dashed blue curves) combinatorial background with one $\eta^{\prime}$ resonance, and (dot-dashed green, dot-dashed black curves) partially reconstructed background with one $\eta^{\prime}$ resonance. The total fit function is shown as the solid blue curve.

\section{Search for $\Lambda_{b}^{0} \rightarrow \Lambda \eta^{(\prime)}[26]$}

A search is performed for the as yet unobserved baryonic $\Lambda_{b}^{0} \rightarrow \Lambda \eta^{\prime}$ and $\Lambda_{b}^{0} \rightarrow \Lambda \eta$ decays using $1.0 \mathrm{fb}^{-1}$ of $p p$ collision data taken by LHCb in 2011 at a centre-of-mass energy of $\sqrt{s}=7 \mathrm{TeV}$ and $2.0 \mathrm{fb}^{-1}$ in 2012 at a centre-of-mass energy of $\sqrt{s}=8 \mathrm{TeV}$. Decays of b-baryons to final states containing $\eta$ or $\eta^{\prime}$ mesons have not yet been observed; however, the branching fractions of the $\Lambda_{b}^{0}$ decays have been estimated to be in the range $(1.8-19.0) \times 10^{-6}$ [27], depending on the model 
used to calculate the hadronic form factors. The interference between Feynman diagrams cancel in such a way that the branching fractions for the $\Lambda_{b}^{0} \rightarrow \Lambda \eta^{\prime}$ and $\Lambda_{b}^{0} \rightarrow \Lambda \eta$ decays are expected to be similar. The decay $B^{0} \rightarrow K_{S}^{0} \eta^{\prime}$ is used as a normalisation channel for the measurement of the branching fractions of the signal decays.

No significant signal is observed above background for the $\Lambda_{b}^{0} \rightarrow \Lambda \eta^{\prime}$ decay, and some evidence is seen for the $\Lambda_{b}^{0} \rightarrow \Lambda \eta$ at the level of 3.0 standard deviations. An upper limit on the absolute branching fraction of the $\Lambda_{b}^{0} \rightarrow \Lambda \eta^{\prime}$ decay is placed using the unified approach [28]. The value is $\mathscr{B}\left(\Lambda_{b}^{0} \rightarrow \Lambda \eta^{\prime}\right)<3.1 \times 10^{-6}$ at $90 \% \mathrm{CL}$. The branching fraction of the $\Lambda_{b}^{0} \rightarrow \Lambda \eta$ decay is $\mathscr{B}\left(\Lambda_{b}^{0} \rightarrow \Lambda \eta\right)=\left(9.3_{-5.3}^{+7.3}\right) \times 10^{-6}$. These results favour the branching fractions calculated using the pole model to estimate the hadronic form factors. In addition, our results are inconsistent with the prediction for $\mathscr{B}\left(\Lambda_{b}^{0} \rightarrow \Lambda \eta^{\prime}\right)$ obtained by neglecting the anomalous contribution to the decay amplitude [27], indicating that a gluonic component of the $\eta^{\prime}$ wavefunction should be present.

\section{First observation of $B_{s}^{0} \rightarrow K_{S}^{0} K^{* 0}$ [29]}

A search for $B_{(s)}^{0} \rightarrow K_{S}^{0} K^{* 0}$ decays is performed using $1.0 \mathrm{fb}^{-1}$ of $p p$ collision data taken by LHCb in 2011 at a centre-of-mass energy of $\sqrt{s}=7 \mathrm{TeV}$. The branching ratios are determined using the $B^{0} \rightarrow K_{S}^{0} \pi^{+} \pi^{-}$decay as a normalisation mode.

Two dimensional extended maximum likelihood fits to the unbinned $K_{S}^{0} K^{+} \pi^{-}$and $K^{+} \pi^{-}$mass distributions are used to determine the event yields for the signal channel (Fig 4), while an independent one-dimensional fit to the $K_{S}^{0} \pi^{+} \pi^{-}$mass distribution is used for the normalisation channel. Decays of $K_{S}^{0}$ mesons are reconstructed in two mutually exclusive categories, long and downstream, depending on whether or not the $\pi$ tracks from the $K_{S}^{0}$ decays have hits in the vertex detector, and these two categories are fitted simultaneously.

The $B_{s}^{0} \rightarrow K_{S}^{0} K^{* 0}$ decay is observed for the first time, with a total significance of 7.1 standard deviations. For the $B^{0} \rightarrow K_{S}^{0} K^{* 0}$ decay, an upper limit is determined. The absolute branching fractions are determined to be $\mathscr{B}\left(B_{s}^{0} \rightarrow K^{0} K^{* 0}\right)=\left(10.9 \pm 2.5(\right.$ stat $) \pm 1.0($ syst $) \pm 0.5\left(f_{s} / f_{d}\right) \pm$ $0.4($ norm $)) \times 10^{-6}$ and $\mathscr{B}\left(B^{0} \rightarrow K_{S}^{0} K^{* 0}\right)=\left(0.15 \pm 0.25(\right.$ stat $) \pm 0.05($ syst $) \pm 0.01($ norm $) \times 10^{-6},<$ $0.64(0.69) \times 10^{-6}$ at $90 \%$ (95\%) CL. where the first uncertainty is statistical, the second systematic, the third due to the ratio of the fragmentation fractions and the fourth due to the uncertainty on the branching fraction of the normalisation decay. These results are in agreement with the theoretical predictions $[18,20,30]$.

\section{Measurement of $B_{s}^{0} \rightarrow \phi \phi \mathbf{B F}[31]$}

In this analysis, the $B_{s}^{0} \rightarrow \phi \phi$ branching fraction is measured using $1.0 \mathrm{fb}^{-1}$ of $p p$ collision data taken by LHCb in 2011 at a centre-of-mass energy of $\sqrt{s}=7 \mathrm{TeV}$ and $2.0 \mathrm{fb}^{-1}$ in 2012 at a centre-of-mass energy of $\sqrt{s}=8 \mathrm{TeV}$. The decay $B^{0} \rightarrow \phi K^{*}(892)^{0}$ is used for normalisation. In addition, a search for the unobserved decay $B^{0} \rightarrow \phi \phi$ is made. This decay is suppressed in the $\mathrm{SM}$, with an expected branching ratio in the range $0.1-3 \times 10^{-8}[8,32,33,34]$. However, the branching ratio could be enhanced in New Physics models. The best limit to date for this mode is from the BaBar collaboration [35], $\mathscr{B}\left(B^{0} \rightarrow \phi \phi\right)<2.0 \times 10^{-7}$ at $90 \%$ confidence level. 

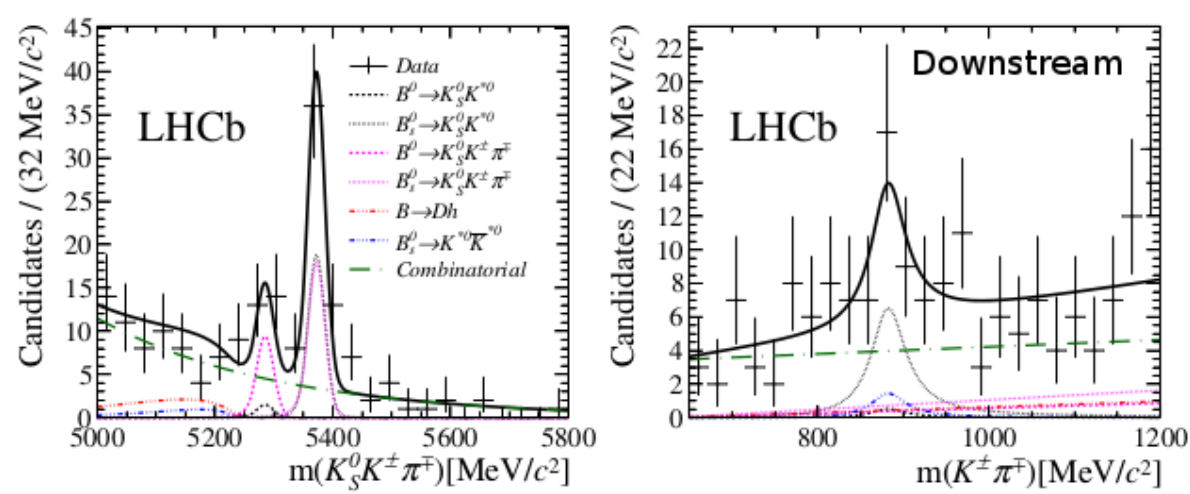

Figure 4: Distribution of $K_{S}^{0} K^{ \pm} \pi^{\mp}$ mass (left) and $K^{ \pm} \pi^{\mp}$ mass (right) for signal candidates in the downstream category, with fit results overlaid. The data are shown as black points with error bars. The overall fit is represented by the solid black line. The $B^{0}$ and $B_{s}^{0}$ signal components are the black short-dashed and dotted lines respectively, while the non-resonant components are the magenta short-dashed and dotted lines. The partially reconstructed backgrounds are the red triple-dotted line $(B \rightarrow D h)$ and the blue triple-dotted line $\left(B_{s}^{0} \rightarrow K^{* 0} \bar{K}^{* 0}\right)$. The combinatorial background is the green long-dash dotted line.

The yields for the signal and normalisation channels are determined from fits to the invariant mass distributions of selected candidates, where $2309 \pm 49$ signal candidates are found. The absolute branching fraction measured is $\mathscr{B}\left(B_{s}^{0} \rightarrow \phi \phi\right)=[1.84 \pm 0.05$ (stat.) \pm 0.07 (syst. $) \pm 0.11\left(\mathrm{f}_{\mathrm{s}} / \mathrm{f}_{\mathrm{d}}\right) \pm$ 0.12 (norm.) $] \times 10^{-5}$. This is in agreement with the measurement made by the CDF collaboration and with theory predictions $[8,36]$.

The data are consistent with no $B^{0} \rightarrow \phi \phi$ contribution. The statistical significance of the fitted $B^{0}$ signal is less than 2 standard deviations, hence a limit on the branching fraction is determined to be $\mathscr{B}\left(B^{0} \rightarrow \phi \phi\right)<3.7 \times 10^{-8}$ at $90 \%$ confidence level. This is tighter than the previous limit set by BaBar [35] and provides a strong constraint on possible contributions from physics beyond the SM to this mode [34].

\section{Conclusions}

The latest results in charmless $B$ decays from LHCb are presented. In the $B_{s}^{0} \rightarrow K^{* 0} \bar{K}^{* 0}$ decay, the low longitudinal polarisation is confirmed, a very large $S$-wave contribution is found and the measurements of the $C P$-violating asymmetries are compatible with the SM. The $B^{0} \rightarrow \rho^{0} \rho^{0}$ decay is observed for the first time and the discrepancy between BaBar and Belle with regard to the different measurements of the longitudinal polarisation is solved. There is no observation of the $B^{0} \rightarrow \rho^{0} f_{0}(980)$ decay. The $B_{s}^{0} \rightarrow \eta^{\prime} \eta^{\prime}$ decay and the $B_{s}^{0} \rightarrow K_{S}^{0} K^{* 0}$ decay are observed for the first time, and also for the first time, an evidence of the $\Lambda_{b}^{0} \rightarrow \Lambda \eta$ decay is detected. Finally, the measurement of the $B_{s}^{0} \rightarrow \phi \phi$ branching fraction is performed, and no evidence of the $B^{0} \rightarrow \phi \phi$ decay is found. All of the presented measurements are consistent with the SM predictions at the current level of precision.

\section{References}

[1] R. Aaij et al. [LHCb Collaboration] JHEP 07 (2015) 166 [arXiv:1503.05362] 
[2] R. Fleischer Phys. Rev. D 60 (1999) 073008 [arXiv:hep-ph/9903540]

[3] M. Ciuchini, M. Pierini and L. Silvestrini Phys. Rev. Lett. 100 (2008) 031802 [arXiv:hep-ph/0703137]

[4] S. Descotes-Genon, J. Matias and J. Virto Phys. Rev. D 76 (2007) 074005 [arXiv:0705.0477]

[5] B. Bhattacharya, A. Datta, M. Duraisamy and D. London Phys. Rev. D 88 (2013) 016007 [arXiv:1306.1911]

[6] R. Aaij et al. [LHCb Collaboration] Phys. Lett. B 709 (2012) 50 [arXiv:1111.4183]

[7] B. Aubert et al. [BaBar Collaboration] Phys. Rev. Lett. 100 (2008) 081801 [arXiv:0708.2248]

[8] M. Beneke, J. Rohrer and D. S. Yang Nucl. Phys. B 774 (2007) 64 [arXiv:hep-ph/0612290]

[9] S. Descotes-Genon, J. Matias and J. Virto Phys. Rev. D 85 (2012) 034010 [arXiv:1111.4882]

[10] A. Datta and D. London Int. J. Mod. Phys. A 19 (2004) 2505 [arXiv:hep-ph/0303159]

[11] R. Aaij et al. [LHCb Collaboration] Phys. Lett. B 747 (2015) 468 [arXiv:1503.07770]

[12] M. Gronau, D. London Phys. Rev. Lett. 65 (1990) 3381

[13] J. Charles et al. Phys. Rev. D 91 (2015) 073007 [arXiv:1501.05013]

[14] A. Bevan et al. Nucl. Phys. Proc. Suppl. 241-242 (2013) 89

[15] B. Aubert et al. [BaBar Collaboration] Phys. Rev. D 78 (2008) 071104 [arXiv:0807.4977]

[16] P. Vanhoefer et al. [Belle Collaboration] Phys. Rev. D 89 (2014) 072008 [arXiv:1212.4015]

[17] R. Aaij et al. [LHCb Collaboration] Phys. Rev. Lett. 115 (2015) 051801 [arXiv:1503.07483]

[18] H.-Y. Cheng and C.-K. Chua Phys. Rev. D 80 (2009) 114026 [arXiv:0910.5237]

[19] J. Sun, G. Zhu and D. Du Phys. Rev. D 68 (2003) 054003 [arXiv:hep-ph/0211154]

[20] A. Ali et al. Phys. Rev. D 76 (2007) 074018 [arXiv:hep-ph/0703162]

[21] A. R. Williamson and J. Zupan Phys. Rev. D 74 (2006) 014003 [arXiv:hep-ph/0601214]

[22] C.-W. Chiang and Y.-F. Zhou JHEP 12 (2006) 027 [arXiv:hep-ph/0609128]

[23] R. Aaij et al. [LHCb Collaboration] Phys. Lett. B 728 (2014) 85 [arXiv:1309.3742]

[24] H.-N. Li and S. Mishima Phys. Rev. D 74 (2006) 094020 [arXiv:hep-ph/0608277]

[25] M. Beneke and M. Neubert Nucl. Phys. B 675 (2003) 333 [arXiv:hep-ph/0308039]

[26] R. Aaij et al. [LHCb Collaboration] JHEP 09 (2015) 006 [arXiv:1505.03295]

[27] M .R. Ahmady, C. S. Kim, S. Oh and C. Yu Phys. Lett. B 598 (2004) 203 [arxiv:hep-ph/0305031]

[28] G. J. Feldman and R. D. Cousings Phys. Rev. D. 57 (1998) 3873 [arxiV:physics/9711021]

[29] R. Aaij et al. [LHCb Collaboration] Submitted to JHEP [arXiv:1506.08634]

[30] F. Su, Y.-L. Wu, C. Zhuang and Y.-B. Yang Eur. Phys. J. C 72 (2012) 1914 [arXiv:1107.0136]

[31] R. Aaij et al. [LHCb Collaboration] Submitted to JHEP [arXiv:1508.00788]

[32] M. Bartsch et al. arXiv:0810.0249

[33] C.-D. Lu, Y.-I. Shen and J.Zhu Eur. Phys. J. C 41 (2005) 311 [arXiv:hep-ph/0501269]

[34] S. Bar-Shalom, G. Eilam and Y.-D. Yang Phys. Rev. D 67 (2003) 014007 [arXiv:hep-ph/0201244]

[35] B. Aubert et al. [BaBar Collaboration] Phys. Rev. Lett. 101 (2008) 201801 [arXiv:0807.3935]

[36] X. Q. Li, G. Lu and Y. D. Yang Phys. Rev. D 68 (2003) 114015 [arXiv:hep-ph/0309136] 